\title{
Can COVID-19 vaccines stop the pandemic?
}

\author{
CS Lau *, MD, FHKAM (Medicine) \\ Department of Medicine, The University of Hong Kong, Hong Kong
}

*Corresponding author: cslau@hku.hk

Hong Kong Med J 2021;27:84-5

https://doi.org/10.12809/hkmj215116

Herd immunity is needed to combat the coronavirus disease 2019 (COVID-19) pandemic. To achieve this, a large proportion of the population must acquire immunisation against severe acute respiratory syndrome coronavirus 2 (SARS-CoV-2), to protect non-vaccinated, immunologically naïve, and immunocompromised individuals. ${ }^{1,2}$ The cost of achieving herd immunity through natural infection by SARS-CoV-2 is unacceptable, as millions of people will succumb to the disease. Therefore, a mass vaccination campaign represents the best countermeasure to reduce the burden of COVID-19 and allow the world to return to normalcy.

Historically, the development of an effective vaccine took years. For example, even the measles vaccine which was found relatively rapidly took 10 years from the discovery of the pathogen to the development of the first vaccine. ${ }^{3}$ With COVID-19 vaccines, the world has witnessed extraordinary progress in just 1 year. As of 2 April 2021, the World Health Organization (WHO) has documented 269 COVID-19 vaccine candidates, with 85 of them in clinical evaluation. ${ }^{4}$ Among these candidates, nine different vaccines across three platformsinactivated viral, adenoviral vector-based DNA, and nucleoside modified mRNA-have been approved for emergency use in 166 countries, areas, or territories. Typically, vulnerable populations, as well as frontline healthcare workers, are the highest priority for vaccination. ${ }^{5}$ Almost all COVID-19 candidate vaccines target the spike proteincomprising a membrane-distal $\mathrm{S} 1$ subunit and a membrane-proximal S2 subunit-that exists in the virus envelope as a homotrimer.

An ideal vaccine should be one that is safe; induces robust immunity and is efficacious in the prevention of infection, symptomatic disease, complications, and transmission; is of high quality; and is stable and easy to distribute, store, and handle for mass administration. The WHO has proposed a number of minimally acceptable attributes and criteria for considerations for the evaluation and prioritisation of COVID-19 vaccines for further development by developers, manufacturers, regulators and funding agencies. Although not all of the nine vaccines currently in use have been enlisted by WHO for emergency use, ${ }^{6}$ all have been evaluated by respective local ministries of health or equivalent agencies and deemed to be safe, efficacious, and of good quality.
In Hong Kong, an inactivated virus vaccine (CoronaVac; Sinovac, China) and an mRNA-based vaccine (Comirnaty; BioNTech, Germany) have been available since the end of February 2021. Although the arrival of COVID-19 vaccines in the city was keenly anticipated, the vaccination rate has been less than desirable thus far. In the first month of the city's vaccination programme, $6.4 \%$ of the total population received a first dose of either vaccine. ${ }^{7}$

For any mass vaccination programmes to be successful, public confidence, which is often undermined by concerns over vaccine safety, is of utmost importance. Regardless of the speed of development of the various COVID-19 vaccines, some adverse events are to be expected. Fortunately, immune-mediated events, such as anaphylaxis, Bell's palsy, Guillain-Barré syndrome, and transverse myelitis, have been rarely reported so far in association with COVID-19 vaccines. However, with millions, if not billions, of people in the world expected to be exposed to new COVID-19vaccines in the near future, different strategies must be deployed to systematically monitor the safety profiles of these vaccines. These strategies include detailed analysis of the safety data of phase I, II, and III clinical trials; regular mandatory post-marketing survey studies to be conducted by the developers; and voluntary reporting by vaccinated subjects and their clinicians. On a public health level, national reporting systems such as the Medicines and Healthcare products Regulatory Agency in the UK and the Vaccine Adverse Event Reporting System in the US are designed to detect early safety problems for licensed vaccines. ${ }^{8}$ Locally, the Department of Health operates a drug safety alert system to capture adverse events following immunisation from the city's medical practitioners, as well as the Hospital Authority and other health agencies. In addition, the Government has commissioned the University of Hong Kong to conduct a prospective surveillance study on adverse events of special interest following vaccination. These data should be regularly reviewed scientifically, and revealed to the public in an open and timely manner to reassure the public of the robust oversight.

On vaccine efficacy, the WHO suggested that a minimum criterion for any acceptable COVID-19 candidate vaccines should be a clear demonstration of at least $50 \%$ point estimate "against disease, severe disease, and/or shedding/transmission endpoints" on a population basis in placebo-controlled efficacy 
trials. ${ }^{9}$ One common misconception that many readers have when interpreting clinical trial reports concerning COVID-19 vaccine efficacies is that these figures could be compared across studies. This is incorrect as there have been no head-tohead studies comparing two or more COVID-19 vaccines. In addition, the various phase III studies reported so far have been conducted in different countries where the COVID-19 situation may vary significantly, involved different study subject groups and employed variable efficacy endpoints, including different clinical and molecular diagnostic criteria for COVID-19 and its severity. Finally, there are no standardised assays for the measurement of SARS-CoV-2 neutralising antibodies which are a key determinant of a vaccine's protection rate.

Observations made in countries with aggressive vaccination policies suggest that mass vaccination is probably effective in controlling COVID-19. Since December 2020, countries such as Israel, the UK, and the US have launched progressive campaigns to vaccinate most of their populations. By the beginning of April 2021, over $60 \%, 46 \%$, and $31 \%$ of the populations of Israel, the UK, and the US, respectively, have received at least one dose of COVID-19 vaccine. Israel has been particularly aggressive with over $55 \%$ of the population fully vaccinated. Encouragingly, all three countries have seen a significant drop in the bi-weekly confirmed COVID-19 cases per million people since midJanuary (Israel: 94\%; UK: 92\%; US: 73\%) and bi-weekly confirmed COVID-19 deaths per million people since the end of January (Israel: 88\%; UK: 95\%; US: $72 \%) .{ }^{10}$ It should be noted that these countries have continued strict non-pharmaceutical interventions including various social distancing policies. Together with a mass vaccination programme, it seems possible to curb the advancement of COVID-19.

So, can vaccination stop the COVID-19 pandemic? This is possible but it will likely take a long time. Worldwide, unfortunately, only $4.7 \%$ of the population have been administered at least one dose of any COVID-19 vaccines so far. ${ }^{5}$ Although the industry has ramped up its efforts in research and development of COVID-19 vaccines, there are too few manufacturers. The increasing production in countries such as Brazil, China, India, and Indonesia may fill the gap but the solution to supply shortage is not yet clear. Coupled with the high cost of vaccines, it is difficult to see how demand could be satisfied or access provided to developing countries, in particular, in the near future. Hong Kong is blessed to have had 15 million doses of vaccines procured for the people so far. It is upon us to get ourselves vaccinated instead of depending on herd immunity. Concerns for vaccine safety and efficacy are understandable but that these are closely monitored locally and internationally, and every effort is being made to reduce vaccineassociated reactions to a minimum. Novel vaccines such as the adenoviral vector and mRNA-based vaccines have their overall effectiveness and safety continuously and carefully monitored.

It is not yet known what proportion of the population we need to vaccinate to achieve herd immunity against COVID-19. A threshold value of $\sim 67 \%$ will be needed assuming that the basic reproductive number $\left(R_{0}\right)$ of the virus is $3 .^{1,2}$ Unless, and until, we reach this threshold, we will not be certain of protection against the epidemic locally and around the world. This also needs to be achieved as quickly as possible with the emergence of SARS-CoV-2 variants which may be resistant to existing vaccines rendering them less efficacious. ${ }^{11}$

"With a fast moving pandemic, no one is safe, unless everyone is safe".11

\section{Author contributions}

The author contributed to the editorial, approved the final version for publication, and takes responsibility for its accuracy and integrity.

\section{Disclosures}

CS Lau is the convener of the Advisory Panel on COVID-19 Vaccines for the Hong Kong SAR Government.

\section{References}

1. Mallory ML, Lindesmith LC, Baric RS. Vaccinationinduced herd immunity: successes and challenges. J Allergy Clin Immunol 2018;142:64-6.

2. Randolph HE, Barreiro LB. Herd immunity: understanding COVID-19. Immunity 2020;52:731-41.

3. Plotkin SA. Vaccines: past, present and future. Nat Med 2005;11(4 Suppl):S5-11.

4. World Health Organization. Draft landscape and tracker of COVID-19 candidate vaccines. Available from: https:// www.who.int/publications/m/item/draft-landscape-ofcovid-19-candidate-vaccines. Accessed 5 Apr 2021.

5. Our World in Data. Coronavirus (COVID-19) vaccinations. Available from: https://ourworldindata.org/covidvaccinations\#vaccine-development-vaccines-approvedfor-use-and-in-clinical-trials. Accessed 5 Apr 2021.

6. World Health Organization. Status of COVID-19 vaccines within WHO EUL/PQ evaluation process. Available from: https://extranet.who.int/pqweb/sites/default/ files/documents/Status_COVID_VAX_01April2021.pdf. Accessed 5 Apr 2021.

7. Department of Health, Hong Kong SAR Government. COVID-19 Vaccination Programme: vaccination dashboard. Available from: https://www.covidvaccine.gov. hk/en/dashboard. Accessed 5 Apr 2021.

8. Castells MC, Phillips EJ. Maintaining safety with SARSCoV-2 vaccines. N Engl J Med 2021;384:643-9.

9. World Health Organization. WHO target product profiles for COVID-19 vaccines. Available from: https://www.who. $\mathrm{int} /$ publications/m/item/who-target-product-profiles-forcovid-19-vaccines. Accessed 5 Apr 2021.

10. Our World in Data. Coronavirus pandemic (COVID-19). Available from: https://ourworldindata.org/ coronavirus\#coronavirus-country-profiles. Accessed 5 Apr 2021

11. World Health Organization. COVAX. Working for global equitable access to COVID-19 vaccines. Available from: https://www.who.int/initiatives/act-accelerator/covax. Accessed 5 Apr 2021. 